\title{
Superimposing Dynamic Range
}
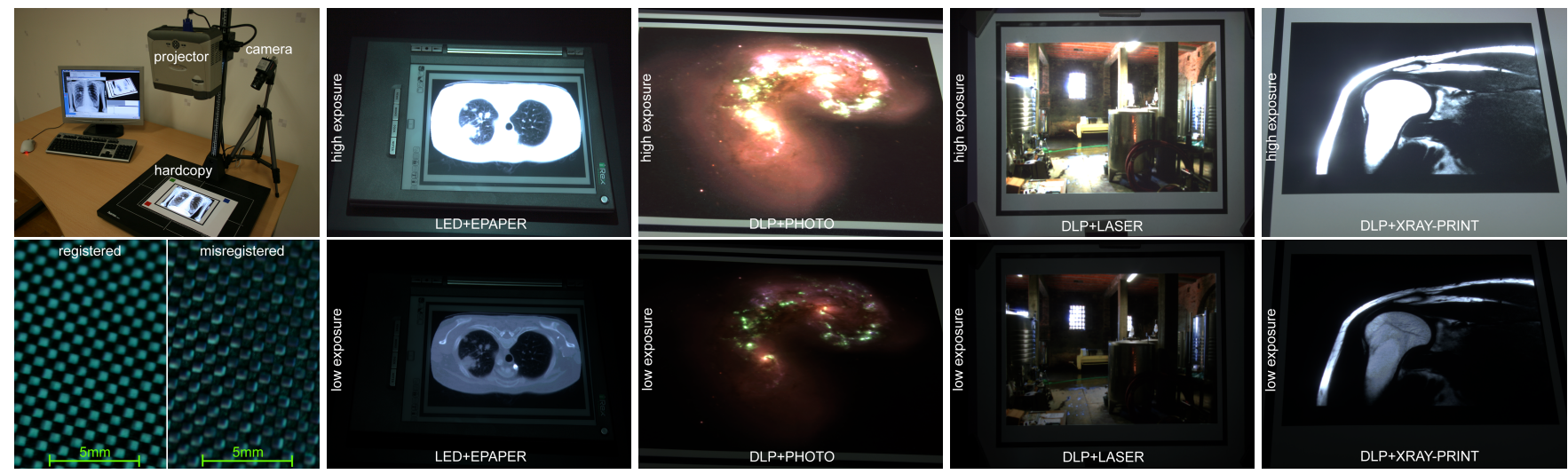

Figure 1: Registering a projector precisely to a hardcopy allows extending contrast, perceivable tonal resolution and color space beyond the capabilities of either hardcopy or projector. From left to right: experimental setup and example for achieved registration precision (projected checker on printed checker with a field size of $0.62 \mathrm{~mm}$ - or 7 cycles per degree (cpd) at $50 \mathrm{~cm}$ viewing distance), low and high exposure photographs of different hardcopies (ePaper display, photographic print, laser print and X-ray print) amplified with LED and DLP projectors.

\section{Abstract}

We present a simple and cost-efficient way of extending contrast, perceived tonal resolution, and the color space of static hardcopy images, beyond the capabilities of hardcopy devices or low-dynamic range displays alone. A calibrated projector-camera system is applied for automatic registration, scanning and superimposition of hardcopies. We explain how high-dynamic range content can be split for linear devices with different capabilities, how luminance quantization can be optimized with respect to the non-linear response of the human visual system as well as for the discrete nature of the applied modulation devices; and how inverse tone-mapping can be adapted in case only untreated hardcopies and softcopies (such as regular photographs) are available. We believe that our approach has the potential to complement hardcopy-based technologies, such as $\mathrm{X}$-ray prints for filmless imaging, in domains that operate with high quality static image content, like radiology and other medical fields, or astronomy.

CR Categories: I.3.3 [COMPUTER GRAPHICS]: Picture/Image Generation-Display algorithms; I.4.0 [IMAGE PROCESSING AND COMPUTER VISION]: General-Image displays; I.4.1 [IMAGE PROCESSING AND COMPUTER VISION]: Digitization and Image Capture;

Keywords: Projector-camera system, hardcopy image, HDR display, luminance quantization, HDR splitting, inverse tone-mapping.

\section{Introduction and Related Work}

Following pioneering work on recovering high dynamic range (HDR) radiance maps from photographs [Mann and Picard 1995; Debevec and Malik 1997], much research has been carried out since then on capturing HDR content as well as on displaying it on low dynamic range (LDR) displays. A large body of tone-mapping techniques, such as the photographic tone reproduction operator [Reinhard et al. 2002] and many others, aim at visually matching the appearance of a tone-mapped HDR image with the observed scene when being displayed on an LDR screen.

It was only recently that HDR displays were introduced which could present content over several orders of magnitude between minimum and maximum luminance. Ledda et al. [Ledda et al. 2003] present a passive stereoscopic HDR viewer that applies two overlaid transparencies for each eye for luminance modulation, and achieves a contrast ratio of 10,000:1. Based on the initial work of Seetzen et al. [Seetzen et al. 2003], active HDR displays were described in [Seetzen et al. 2004] that modulate images displayed on an LCD panel with a locally varying background illumination. This is either produced by a lower-resolution LED panel, or by a higher-resolution DLP projector. A contrast ratio of over 50,000:1 together with a peak luminance of $2,700 \mathrm{~cd} / \mathrm{m}^{2}$ (for the projector-based backlight) and $8,500 \mathrm{~cd} / \mathrm{m}^{2}$ (for the LED-based backlight) were reported. Rosink et al. [Rosink et al. 2006] describe an HDR display prototype that utilizes two parallel-aligned (5 Mpixel) LCD panels which together enable a per-pixel contrast of 3,000-100,000:1 (depending on the viewing angle) together with a peak luminance of $1,000 \mathrm{~cd} / \mathrm{m}^{2}$. A color LCD backlight modulator behind a monochrome front panel allows one to adjust the exact white point of the display. Pavlovych et al. [Pavlovych and Stuerzlinger 2005] proposed an HDR projector that modulates the image path (i.e., after image generation) with a low-resolution monochrome LCD panel and a set of lenses placed in front of a regular DLP projector's objective. It attains an ANSIcontrast of 708:1 and a peak luminance of $425 \mathrm{~cd} / \mathrm{m}^{2}$. Kusakabe et al. [Kusakabe et al. 2006] also describe an HDR projector that modulates the illumination path (i.e., between light source and image generator) with three (RGB) integrated low-resolution LCoS panels before a subsequent luminance modulation occurs with a 

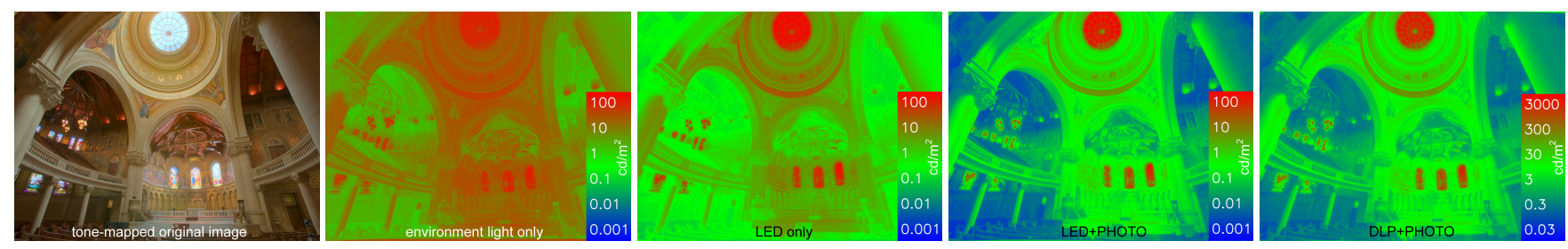

Figure 2: Measured contrast of tone-mapped HDR image as photographic print under environment light and as projection on a white screen. Split HDR content displayed with LED+PHOTO and DLP+PHOTO - leading to contrast enhancements of 2-3 orders of magnitude.

high-resolution monochrome LCoS panel. Similar approaches are explained by Damberg et al. [Damberg et al. 2007] who achieve a simultaneous contrast of 2,695:1. A variety of inverse tone-mapping techniques are now being developed, such as Banterle et al. [Banterle et al. 2006] and others, to convert existing LDR content into an HDR format to be viewed on such devices.

All of these approaches share three common properties: These are firstly that they apply a transmissive image modulation (either through transparencies or LCD/LCoS panels) and consequently suffer from a relatively low light-throughput (e.g., regular color / monochrome LCD panels transmit less than 3-6\% / 15-30\% of light) and therefore require exceptionally bright backlights. Secondly, with Rosink et al. [Rosink et al. 2006] as an exception, one of the two modulation images is of low-resolution and blurred in order to avoid artifacts such as moiré patterns due to the misalignment of two modulators, as well as to realize acceptable frame-rates. Thus, high contrast values can only be achieved in a resolution of the low-frequency image. Thirdly, since one of the two images is monochrome (mainly to reach a high peak luminance), only luminance is modulated, while chrominance modulation for extending the color space is in some cases considered future work.

In this paper we present a simple and low-cost method of viewing static HDR content based on reflective image modulation. We project images onto hardcopies, such as photographs, X-ray prints, or electronic paper (ePaper) so as to boost contrast, perceivable tonal resolution, and color space values beyond the potential of either hardcopies (when viewed under environment light) or projectors (when projecting onto regular screens) alone. Figure 1 illustrates examples thereof. We do not intend to compete with interactive HDR displays, but rather offer an "everybody can do" alternative for domains that operate with static image content, such as radiology and other medical fields, or astronomy. Yet, electronic paper will allows for interactive visualizations. In contrast to related projector-camera approaches that perform radiometric or photometric compensations, such as Grossberg et al. [Grossberg et al. 2004] and others, our goal is to extend the dynamic range on hardcopy images rather than fitting arbitrary image content into the limited contrast range of nonoptimized projection surfaces with varying reflectance properties. In our experiments, we achieved contrast ratios of over 45,000:1 with a peak luminance of more than $2,750 \mathrm{~cd} / \mathrm{m}^{2}$, could technically re-produce more than 620 perceptually distinguishable tonal values (approximately $85 \%$ of all theoretically possible JND steps). Furthermore, we attained color space extensions of up to factor 1.4 (compared to a regular projections) or factor 3.3 (compared to regular hardcopy prints). Thereby, the hardcopy resolution can be several thousand dots per inch, while luminance and chrominance are modulated with a registration error of less than $0.3 \mathrm{~mm}$.

\section{Geometric Registration and Photometric Calibration}

A precise geometric registration between projector, hardcopy and its softcopy (i.e., a digital version of the hardcopy image) is essen- tial. We have implemented three automatic registration methods for different situations and these are the following:

Homography. The homography between projector and camera over the common table plane is measured initially. Hardcopies are printed with an additional frame that robustly allows one to detect its corner points in the camera perspective. The homography matrix together the corner points allows the warping of all camera pixels into the projector perspective and registering them precisely to the corresponding pixels of its softcopy. Homographies require the hardcopies to be fully planar. This is achieved by clamping them flat on the table plane or by applying professional vacuum tables.

The additional printed frame as well as the preconditioned planarity of the hardcopy, represent constraints that might only be met by some applications while others require more flexibility. Images printed on photographic paper are usually never perfectly flat. Furthermore, parts of a full-scale image may be cut away during the reformatting process of the printer, and image portions at the border do not appear in the hardcopy. Thus, a simple registration via homography and corner points will not be sufficient. Assuming that the hardcopy is arbitrarily shaped, but does not contain geometric (but possibly radiometric) discontinuities, we would in such cases then apply the following registration techniques:

Structured Light. Structured light techniques, such as Gray codes, can be used for measuring the pixel correspondences between camera and projector over a non-planar hardcopy surface. However, this must be robust for non-uniformly colored and dark surface portions that absorb a large amount of projected light. Furthermore, we strive for a method that requires capturing a minimum number of images for registration, so as to both speed-up the calibration process, as well as to prevent it from over-demanding mechanical parts, in case digital SLR cameras are used. Our implementation requires the capture of three images only: Two images display horizontal and vertical grid lines as well as a color-coded, absolute reference point in the grid center. The third image captures the hardcopy under a projected white illumination. The two grid images are divided by the white-light image for normalization, and the results are thresholded and binarized. The lines, as well as the reference point, are recovered through labeling and line tracing; and intersections of connected line segments are detected. The intersection points are triangulated relative to the absolute coordinates of the reference point and intermediate projector-camera correspondences are interpolated. As a result, the precision of this techniques depends mainly on the adjusted grid resolution and on the degree of curvature of the hardcopy. For homography and structured light, misregistrations are in general below $0.3 \mathrm{~mm}$ (cf. figure 1 ).

Matching Image Features. Feature points are detected within the captured camera image of the hardcopy, and are matched against image features found within the softcopy [Lepetit and Fua 2006]. All matched feature points are triangulated, and missing correspondences inside and outside the convex hull of the constructed triangle mesh are interpolated and extrapolated respectively. The resulting look-up table provides pixel correspondences between hardcopy and 
softcopy that can be used in combination with the projector-camera correspondences (determined either through homography and corner points, or measured via structured light) for relating projector pixels with corresponding softcopy pixels. Although feature-based registration techniques are quite invariant to photometric differences between a captured hardcopy and digital softcopy, their precision depends very much on the number and on the distribution of detected feature points - and consequently on the image content.

Photometric Calibration. All techniques described in this paper require linearized transfer functions of projector and camera. The camera has been calibrated with a spectroradiometer in order to deliver correct luminance and chrominance values. Furthermore, the non-linear light drop-off and the contribution of the environment (including the projector's black level) on the projection surface, as well as the color mixing taking place between projector and camera have to be measured and compensated for all projected and captured images. In order to achieve this, we apply well-established photometric calibration techniques for projector-camera systems. Describing further details regarding these techniques is out of the scope of this paper. They are summarized in [Brown et al. 2005; Bimber et al. 2007]. If projectors with pulse-width modulation are applied (such as DLP or GLV), the projection's refresh time must always be an integer multiple of the camera's exposure time, so as to ensure the correct integration over all colors and intensities. For displaying purposes however, an arbitrary refresh rate can be chosen. Information on the photometric correction of hardcopy devices will be provided together with individual rendering techniques in the following sections.

\section{Rendering Techniques}

In this section, we explain how existing HDR content can be split for linearized hardcopy devices and projectors, how luminance quantization can be optimized with respect to the non-linear response of the human visual system and the discrete nature of the applied devices, and how inverse tone-mapping can be adapted in case only untreated hardcopies and softcopies (such as regular photographs) are available.

\subsection{Splitting High Dynamic Range Content}

Splitting a given high dynamic range image $\left(I_{H D R}\right)$ into a low dynamic range pair $\left(I_{A}, I_{B}\right)$ consisting of projected image and printed or displayed hardcopy can be achieved with:

$$
\begin{gathered}
I_{A}=T M_{A B}\left(I_{H D R}\right)^{\gamma \frac{a}{a+b}} \\
I_{B}=T M_{A B}\left(I_{H D R}\right)^{\gamma} / T_{A}\left(I_{A}\right),
\end{gathered}
$$

where $A$ is the device (i.e., either projector or hardcopy device, such as a printer or an ePaper display) with a possibly significantly lower image quality compared to the other device $(B)$, with respect to tonal and spatial resolution, color space, banding and dithering effects. $T M_{A B}$ is an (inverse) tone-mapping operator that maps $I_{H D R}$ to the tonal resolution and the color gamut that result from the modulation of $A$ and $B$ (section 4 provides examples for combinations of different projectors and hardcopies). A gamma correction can be applied optionally before the mapped HDR content is split relative to the individual bit depths $(a, b)$ of both devices. Thus, equation 1 computes the image for the lower quality device with bit depth $a$, while equation 2 compensates for artifacts with $I_{B}$ that are generated when displaying or printing $I_{A}$. Thereby, $T_{A}$ is the linearized transfer function of $A$ that allows simulating the appearance of $I_{A}$ with respect to $A$ 's actual color space, tonal and spatial resolution, and possible spatial artifacts produced by banding or dithering. An example is shown in figure 2 .
In the following, we summarize several splitting conventions that optimize the image quality depending on the capabilities of both devices:

- General. Always split for the device $(A)$ with lower image quality and compensate with the higher quality device $(B)$. This allows for compensating artifacts in $I_{A}$ with $I_{B}$ as efficiently as possible.

- Both Color. If $A$ and $B$ can both display colors, then splitting and compensation are both carried out in RGB space (instead of in luminance space followed by a recombination) to avoid clipping artifacts during compensation.

- Low-Quality Gray. If $A$ can display gray scales only, and $B$ displays colors, then splitting is carried out in luminance space while compensation is processed in RGB space to achieve the desired original colors and intensities (as in [Seetzen et al. 2004; Trentacoste et al. 2007]).

- Low-Quality Color. If $A$ displays colors and $B$ displays gray scales only, then splitting and compensation are both carried out in luminance space, while $I_{A}$ is converted back to RGB space before being displayed. Only a luminance compensation is possible in this case, while colors are approximated and chromatic artifacts remain uncompensated.

- Both Gray. If $A$ and $B$ both display gray scales only, then splitting and compensation are carried out both in luminance space.

All of these techniques require the transfer functions of the projector and hardcopy device to be linear. In addition to linearizing the projector response as indicated in section 2 , we measure the full (color and intensity) transfer function of the hardcopy device by printing or displaying and capturing all possible hues and tonal values during the one-time device calibration process. For an 8-bit RGB photo printer ${ }^{1}$, for instance, all $2^{24}$ values can be spatially encoded and printed on four letter-sized color charts. These charts that are captured with a linearized high-resolution camera under a uniform white projector illumination are rectified, indexed; and their entries are sampled, smoothened, and stored in a look-up table. For linearization, this look-up table is reversed. Multiple entries are averaged and missing values inside the convex hull of the sampled points are interpolated. Missing values outside the convex hull are mapped to their closest valid entries. Note that these look-up tables sample only the color and intensity transfer up to scale, while for $T_{A}$ in equation 2 one should consider spatial image transfer effects, such as banding and dithering. We can compute these effects based on known dithering and sampling functions, rather than measuring them. For reasons of precision, we store and apply the full look-up tables instead of separating individual color channels and fitting them into a set of analytical functions.

If the projector and hardcopy device are both linear and share the same transfer properties (tonal resolution, spatial resolution, color space, etc.), then HDR splitting and registration between soft- and hardcopy becomes unnecessary. In this case, the hardcopy can display $\sqrt{\left(I_{H D R}\right)^{\gamma}}$, while a high-resolution, linear photograph thereof can be projected back without modification (except a possible but constant intensity scaling that takes the $f$-stop settings of the camera into account). This delivers acceptable results if the linearized camera response does not significantly reduce the image quality. Only a registration between camera and projector is required. This option even leads to reasonable results if the transfer properties of both devices are not exactly the same, but very similar and the hardcopy device has a better image quality than the projector. However, spatial

\footnotetext{
${ }^{1}$ such as the Kodak System 88 which applies an 8-bit RGB LCD panel for light modulation during development
} 
image transfer effects (banding and dithering) are not compensated. It is valid for color images projected onto color hardcopies (color on color), as well as for gray on color, and gray on gray cases, but it fails for color on gray situations.

\subsection{Luminance Quantization}

As for other HDR display approaches, the modulation occurring for the projection and hardcopy leads to a large number of physically produceable luminance levels. Due to the non-linear response of the human visual system however, not all are perceptually distinguishable. The number of discernible luminance levels (Just Noticable Difference, or JND, steps) increases with a rising peak luminance of the display [Seetzen et al. 2004]. Since an exact representation with
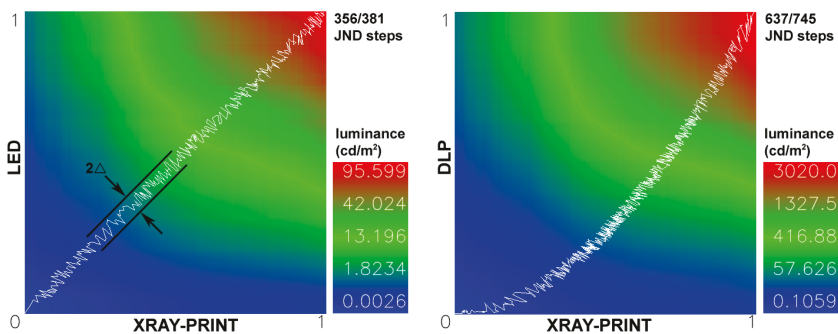

Figure 3: Luminance values in normalized gray scale space and selected JND steps (white) for combinations (see section 4): $L E D+X R A Y-P R I N T$ and DLP+XRAY-PRINT with $\Delta=0.025$.

guaranteed distinguishable luminance levels is essential for several professional applications, such as with many medical visualizations, images should be converted to a perceptually linear JND space, rather than being presented in a physically linear luminance space. Ghosh et al. [Ghosh et al. 2005], for instance, describe perceptually linear transfer functions for volume rendering on HDR displays. However, the technically achievable luminance space of such displays is discretized and holds a quantization challenge, in that on the one hand, selected JND steps may not be achieved exactly, since they do not map to a produceable luminance levels. This is especially the case if both modulators (in our case, projector and hardcopy device) are independently linearized, leading to a reduction of tonal values per se in each individual channel, or have a small local tonal resolution. On the other hand, many similar luminance levels can be approached with more than one modulation combination. This raises the following question: How can selected JND steps be optimally mapped to individual modulator responses, in such a way that a maximum of all (theoretically) possible JND steps is technically achieved and that the combination of both modulators' transfer functions, which produce the selected JND steps, remains as monotone as possible. The second condition is important to avoid visual artifacts in case of slight misregistrations, significant differences in modulator resolution, or imprecision occurring in their measured transfer functions.

For displaying gray scale content, we solved this problem by sampling the reflected luminance values of all $2^{h}$ hardcopy gray scales (from the transfer look-up table explained in 3.1), and of all $2^{p}$ projector gray scales (projected onto a flat, white hardcopy sample). Their multiplication leads to the corresponding luminance values for all $2^{h+p}$ gray scale combinations. Assigning the normalized gray scales to $x$ (hardcopy) and $y$ (projector) coordinates $(0 \leqslant x, y \leqslant 1)$, our goal is to fit $y=x^{\sigma}$ with the following objective:

$$
\begin{array}{r}
\max \left(\left|\bigcup_{j=0}^{n}\left\{s_{j} \mid \min _{s_{j} \in C_{j}}\left(L_{s_{j}}-L_{j}\right)^{2}\right\}\right|\right), \\
C_{j}=\left\{c \mid \Delta_{c}<\Delta, L_{j} \leqslant L_{c}\right\},
\end{array}
$$

where $j=0 . . n$ indexes each individual JND step (with desired luminance $L_{j}$ ) that can be derived from given black level and peak luminance. We apply the luminance quantization function described in [Mantiuk et al. 2005; Mantiuk et al. 2004], since it is defined for our luminance range. Thereby, $L_{0}$ is equivalent to the lowest black level reflection of the projector. For each (theoretically) possible JND step $(j)$ we choose a set $\left(C_{j}\right)$ of gray scale candidates $\left(c \in C_{j}\right)$ that leads to re-produceable luminance levels larger than or equal to JND step $j$, and whose shortest $(x, y)$-distances $\left(\Delta_{c}\right)$ to $y=x^{\sigma}$ are not larger than a predefined maximum $(\Delta)$. From each $C_{j}$, we select the candidate $s_{j} \in C_{j}$ that approaches $L_{j}$ best. Fitting $y=x^{\sigma}$ while maximizing the number of technically achievable JND steps, results in one optimal set of projector and hardcopy gray scales for each JND step that satisfies our conditions. These are the gray scales that belong to the selected samples $s_{j}$ for each JND step $j$ at the optimal $\sigma$. Figures 3 and 6 illustrate examples.

For displaying color content, the luminance of the original RGB values are scaled for the projector and hardcopy with the corresponding (normalized) gray scales that have been selected for these components (cf. figure 6-bottom row for a color on gray example).

\subsection{Inverse Tone-Mapping}

This section discusses the situation where no explicit HDR softcopy is available, but where instead only a non-linear hardcopy and possibly a non-linear LDR softcopy exist. These could be an ordinary and untreated photographic print and its digital counterpart. Capturing high-resolution HDR images of the hardcopy under a full white and under a full black projection, results in the local maximum $\left(I_{\max }\right)$ and minimum $\left(I_{\min }\right)$ reflectance at every single point on the hardcopy. Converting this data to local luminance values, the global maximum $\left(\bar{L}_{\max }\right)$ as well as the global minimum $\left(\bar{L}_{\min }\right)$ can be determined.

If an LDR softcopy is available, then one option is to apply an inverse tone-mapping operator to approximate an HDR representation $\left(I_{H D R}\right)$, register it to the hardcopy and apply equation 4 to compute the projected image:

$$
I_{P}=\left(I_{H D R}-I_{\min }\right) /\left(I_{\max }-I_{\min }\right)
$$

We apply the inverse tone-mapping operator described by Banterle et al. [Banterle et al. 2006] (which reverses the global version of the photographic tone reproduction operator [Reinhard et al. 2002]) for this (cf. figure 1 for an example).

If no LDR softcopy is available, then two possibilities exist. For both cases, only a projector-camera registration is necessary. In one option, we can use $I_{\max }$ directly as input for the inverse tonemapping operator. This however, can lead to a significant loss in image quality due to the limited camera response, as well as due to the non-linear and restricted color reproduction capabilities of the hardcopy device. A better solution is to reconstruct the colors of the original LDR softcopy by applying the inverse transfer function of the hardcopy device to $I_{\max }$. In our case, this is equivalent to indexing the reverse transfer look-up table described in section 3.1. Figure 4 illustrates two reconstruction examples.

In [Akyüz et al. 2007] it was argued that from a perceptual point of view, simple scaling transformations can yield similar results as sophisticated tone-mapping operators (and sometimes even outperform them). We can adapt their scaling transformation for our purpose:

$$
L_{H D R}=\left(\bar{L}_{\max }-\bar{L}_{\min }\right)\left(\frac{L-L_{\min }}{L_{\max }-L_{\min }}\right)^{\gamma}+\bar{L}_{\min },
$$

whereby -as above- $L$ can either be the luminance values of $I_{\max }$ or the luminance values of the reconstructed softcopy, and $L_{\max }$, $L_{\min }$ are the global maximum and minimum of $L$. After converting $L_{H D R}$ to RGB $\left(I_{H D R}\right)$, equation 4 can be used for estimating the 


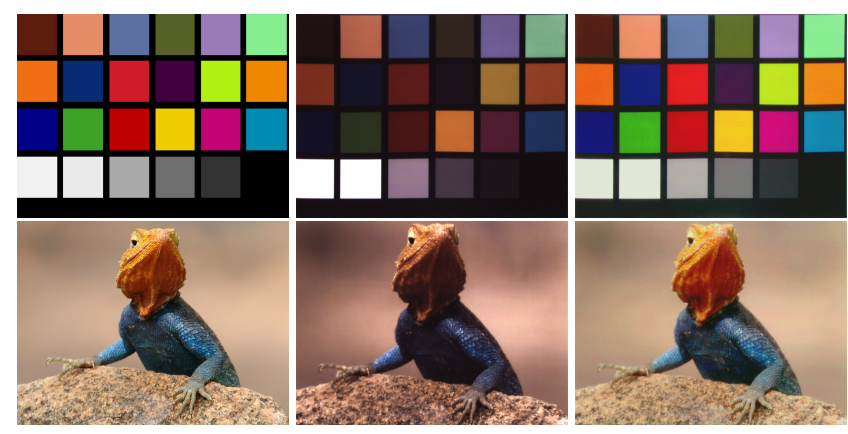

Figure 4: Color reconstruction of Macbeth samples (top) and digital image (bottom) developed on photographic paper with a Kodak System 88 digital lab system. The original (left), rectified camera image of photo without (center) and with (right) reconstructed colors. The reconstructions show a $4 \%$ chrominance deviation to original in CIE $L * a * b *$ space.

projection image. Note, that since we do not assume a linear transfer function of the hardcopy device, a minimum of clipping during compensation with equation 4 is ensured if $\gamma$ in equation 5 equals the gamma of the hardcopy device's transfer function. As for the cases above, a registration of softcopy and hardcopy is not necessary. Only a registration between projector and camera is required.

\section{Results}

Table 1 presents measurement results for different combinations of projectors and hardcopy devices. The color space extensions

\begin{tabular}{|c|c|c|c|c|c|c|}
\hline & parameters & PHOTO & XRAY-PRाNT & LASER & EPAPER & SCREEN \\
\hline \multirow{4}{*}{ DLP } & CIE $x y$ coverage $(\%)$ & 36.4 & 38.1 & 37.5 & n.a. & 26.8 \\
\hline & contrast ratio & $45,679: 1$ & $28,516: 1$ & $15,595: 1$ & $6,458: 1$ & 654:1 \\
\hline & peak luminance $\left(\mathrm{cd} / \mathrm{m}^{2}\right)$ & 2,750 & 3,020 & 3,100 & 1,440 & 3,100 \\
\hline & \#JND / \#JND max & $628 / 743$ & $637 / 745$ & $623 / 738$ & $329 / 644$ & \\
\hline \multirow{4}{*}{ LED } & CIE $x y$ coverage $(\%)$ & 58.6 & 59.4 & 59.2 & n.a. & 55.7 \\
\hline & contrast ratio & $61,854: 1$ & $36,167: 1$ & $23,618: 1$ & $8,458: 1$ & 945:1 \\
\hline & peak luminance $\left(\mathrm{cd} / \mathrm{m}^{2}\right)$ & 87.1 & 95.6 & 97.3 & 43.2 & 97.3 \\
\hline & \#JND / \#JND max & $345 / 373$ & $356 / 381$ & $349 / 380$ & $201 / 286$ & $169 / 335$ \\
\hline \multirow{4}{*}{ ENVIRONMENT } & CIE $x y$ coverage $(\%)$ & 10.9 & 10.5 & 9.8 & n.a. & n.a. \\
\hline & contrast ratio & $73.3: 1$ & $40.4: 1$ & $32.2: 1$ & $9.79: 1$ & n.a. \\
\hline & peak luminance $\left(\mathrm{cd} / \mathrm{m}^{2}\right)$ & 67.1 & 70.3 & 77.2 & 37.7 & 77.2 \\
\hline & $\# J N D / \# J N D$ max & $128 / 255$ & $126 / 247$ & $118 / 250$ & $16 / 155$ & n.a. \\
\hline
\end{tabular}

Table 1: Results for combinations of different projectors and hardcopy devices.

for the various combinations is additionally illustrated in figure 5. Our experimental hardware configuration is shown in figure 1. The hardcopy is placed on a tray which can optionally be tilted slightly for specular photographs to direct highlights away form the observer. In particular, we evaluated the following devices: A

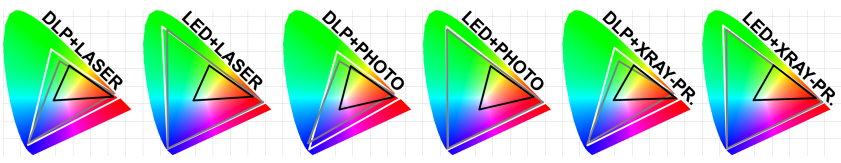

Figure 5: Coverage of CIE xy chromaticity space. Here, hardcopy papers under environment light (black), projectors on white screen (gray), and combinations (white).

300 dpi Kodak System 88 professional digital lab system for photographs (PHOTO) together with Kodak Royal N photo paper, a 2400dpi Xerox WorkCentre 7655 professional printer used for medical data (XRAY-PRINT), a 1200dpi Samsung CLP-510N consumer color laser printer (LASER), a XVGA/160dpi iRex iLiad ePaper display $(E P A P E R)$, a SVGA MITSUBISHI PK20 LED projector
$(L E D)$, and a XGA Optoma DX733 DLP projector $(D L P)$. For calibration and measurements, we alternatively apply one of the two CMOS cameras, these being a 10.1Mpixel Canon EOS Digital Rebel XTi consumer digital SLR camera and a QXGA ARTRAY ARTCAM 300MI professional image processing camera. Both have been linearized and calibrated with a spectroradiometer. While the ARTCAM is faster, the EOS offers a higher resolution and more $f$-stops. For taking measurements under environment light ( $E N$ VIRONMENT), a 20W halogen lamp was used, while for regular screen projections (SCREEN) a blank sheet of the same paper type as for $L A S E R$ was used. In most combinations, contrast ratio, color space coverage, and number of achievable JND steps versus theoretically possible JND steps for the corresponding luminance range (\#JND/\#JNDmax) are boosted significantly with two exceptions: The LEDs of the LED projector are already quite saturated, which leads to only minor extensions of the color space. It is also relatively dim, and a large number of JND steps are not possible due to its low peak luminance. However, it enables the largest overall contrast ratio. Due to the low native contrast and tonal resolution (only 16 gray scales) of the ePaper display, the overall contrast and number of JND steps that were achieved with it were roughly less than half compared to the other combinations. However, it allows for displaying interactive content.

\section{Discussion}

Specialized printing technologies that support filmless imaging hold several potentials for many medical and other professional domains. Compared with conventional hardcopy media, such as X-ray film, they offer significant cost reductions, longer durability (less light sensitivity) and colored visualization usage. They provide near diagnostic quality and a much higher spatial resolution than possible with most interactive displays, but do not reach the high contrast, luminance and perceivable tonal resolution of, for instance, X-ray film viewed with light boxes. Our approach represents a cost-efficient add-on for such hardcopy technologies that allows extending contrast, tonal resolution and color space beyond their capabilities.

Compared with most existing interactive HDR displays, we support near distance viewing at a contrast resolution of up to $7 \mathrm{cpd}$ (given our current registration precision and assuming a viewing distance of $50 \mathrm{~cm}$ ). Due to scattering of light in the eye, the perceived local contrast is reduced, depending mainly on the adaptation luminance and on the spatial frequency of the observed content. Referring to the optical transfer function of the eye described in [Mantiuk et al. 2005], we can still achieve a perceived local contrast of $49 \%-69 \%$ of the physical contrast at a spatial resolution of $7 \mathrm{cpd}$ and an adaptation luminance of $0.06 \mathrm{~cd} / \mathrm{m}^{2}-2,750 \mathrm{~cd} / \mathrm{m}^{2}$ (i.e., 22,000 - 31,000:1 of 45,000:1). It is also reported in [Mantiuk et al. 2005] that the contrast sensitivity (CS) for an adaptation luminance above 1,000 $\mathrm{cd} / \mathrm{m}^{2}$ is maximal at this resolution, and that the CS-peak only shifts to lower frequencies with a decreasing adaptation luminance. A high contrast resolution seems to be important for HDR displays applied for medical visualizations. Therefore, initial displays that achieve a pixel-precise backlight modulation are being developed [Rosink et al. 2006]. However, a double modulation through transmissive layers, such as LCD panels, will always suffer from extremely low light throughput and an adequately high peak luminance that produces many distinguishable tonal values, may be difficult to achieve. A reflective modulation, such as in our case, is more efficient here. Yet, it is also important to ensure that computed JND steps can truly be achieved, which is not necessarily the case due to a discretization of the modulators' responses. Simply mapping to nearest neighbors might lead to indistinguishable selections and/or to a non-maximized number of produced JND steps. We hope that our luminance quantization technique stimulates the development of emerging rendering techniques for interactive HDR displays. 

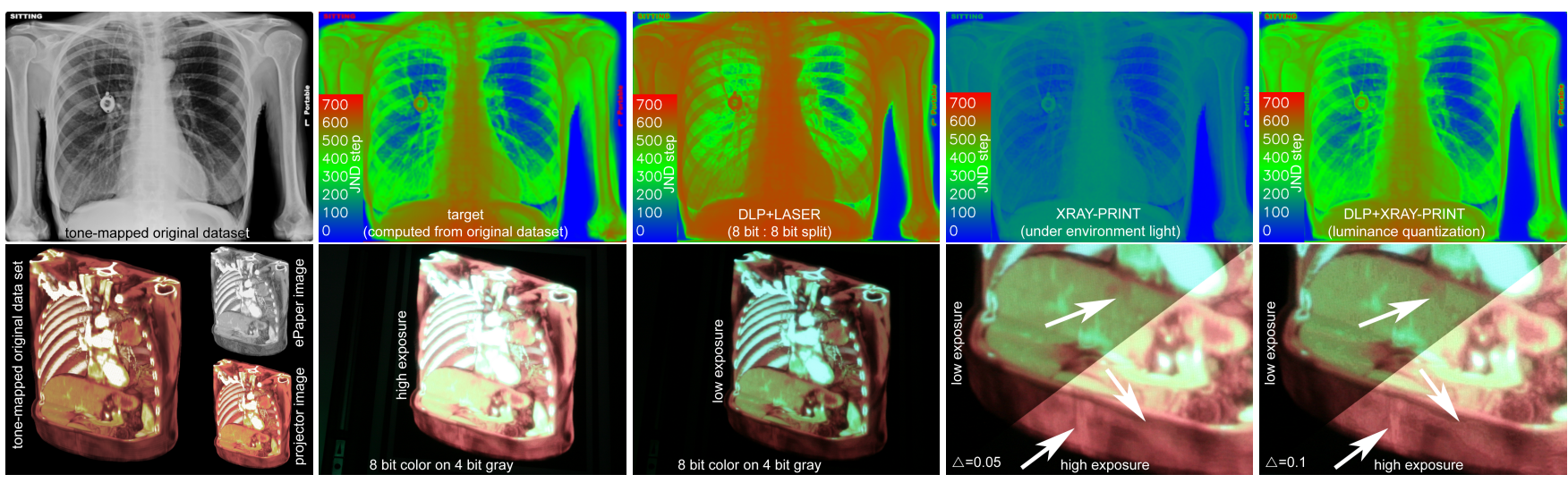

Figure 6: Top row: Comparing simulated target JND steps with measured JND steps of dissimilar modulation combinations (photographs rectified). Splitting leads to perceptually non-linear results, while plain X-ray prints support only a small number of JND steps. Bottom row: $L E D+E P A P E R$ color on gray - reviling artifacts in close-up photographs if a too high $\Delta$ is chosen for luminance quantization (right).

\section{References}

AkyÜZ, A. O., Fleming, R., Riecke, B. E., Reinhard, E., AND BÜlthofF, H. H. 2007. Do hdr displays support ldr content?: a psychophysical evaluation. In Proc. ACM Siggraph, vol. $26,38.2-38.7$.

Banterle, F., Ledda, P., Debattista, K., And Chalmers, A. 2006. Inverse tone mapping. In Proc. Conference on Computer Graphics and Interactive Techniques in Australasia and Southeast Asia, 349-356.

Bimber, O., Iwai, D., Wetzstein, G., And Grundhoefer, A. 2007. The Visual Computing of Projector-Camera Systems. In Proc. Eurographics (State-of-the-Art Report), 23-46.

Brown, M., Majumder, A., And YAng, R. 2005. Camera Based Calibration Techniques for Seamless Multi-Projector Displays. IEEE Trans. on Visualization and Computer Graphics 11, 2, 193 206.

Damberg, G., Seetzen, H., Ward, G., Heidrich, W., AND WHITEHEAD, L. 2007. High dynamic range projection systems. In Society of Information Displays Symposium Digest, vol. 38, 4-7.

DebeVec, P. E., AND Malik, J. 1997. Recovering high dynamic range radiance maps from photographs. In Proc. ACM Siggraph, 369-378.

Ghosh, A., Trentacoste, M., And Heidrich, W. 2005. Volume rendering for high dynamic range displays. In Proc. EG/IEEE VGTC Workshop on Volume Graphics, 91- 231.

Grossberg, M., Peri, H., Nayar, S., And Belhumeur, P. 2004. Making One Object Look Like Another: Controlling Appearance using a Projector-Camera System. In Proc. IEEE Conference on Computer Vision and Pattern Recognition, vol. I, 452-459.

Kusakabe, Y., KanaZawa, M., NoJiri, Y., FURUYA, M., AND Yoshimura, M. 2006. YC-separation Type Projector with Double Modulation. In Proc. International Display Workshop, 1959-1962.

LeddA, P., WARD, G., AND Chalmers, A. 2003. A wide field, high dynamic range, stereographic viewer. In Proc. Conference on Computer Graphics and Interactive Techniques in Australasia and Southeast Asia, 237-244.
LePETIT, V., AND FUA, P. 2006. Keypoint recognition using randomized trees. IEEE Trans. on Pattern Analysis Machine Intelligence 28, 9, 1465-1479.

MANN, S., AND PICARD, R. 1995. Being 'undigital' with digital cameras: Extending dynamic range by combining differently exposed pictures. In Proc. IS\&T 46th annual conference, 422428.

Mantiuk, R., KrawczyK, G., Myszkowski, K., AND SeIdel, H.-P. 2004. Perception-motivated high dynamic range video encoding. In Proc. ACM Siggraph, vol. 23, 733-741.

Mantiuk, R., Daly, S. J., Myszkowski, K., And Seidel, H.-P. 2005. Predicting visible differences in high dynamic range images - model and its calibration. In Proc. IS\&T/SPIE's Annual Symposium on Electronic Imaging, vol. 5666, 204-214.

Pavlovych, A., And Stuerzlinger, W. 2005. A HighDynamic Range Projection System. In Proc. SPIE annual meeting, vol. 5969.

Reinhard, E., Stark, M., Shirley, P., ANd Ferwerda, J. 2002. Photographic tone reproduction for digital images. In Proc. ACM Siggraph, vol. 21, 267-276.

Rosink, J., Chestakov, D., Rajae-Joordens, R., Albani, L., ARENDS, M., AND HeETEN, G. 2006. Innovative lcd displays solutions for diagnostic image accuracy. In Proc. Radiological Society of North America annual meeting, electronic abstract.

Seetzen, H., Whitehead, L. A., And Ward, G. 2003. A high dynamic range display using low and high resolution modulators. In Proc. Society for Information Display, 1450-1453.

Seetzen, H., Heidrich, W., Stuerzlinger, W., Ward, G., Whitehead, L., Trentacoste, M., Ghosh, A., And Vorozcovs, A. 2004. High dynamic range display systems. In Proc. ACM Siggraph, 760-768.

Trentacoste, M., Heidrich, W., Whitehead, L., Seetzen, H., AND WARD, G. 2007. Photometric image processing for high dynamic range displays. J. Visual Communication and Image Representation 18, 5, 439-451. 